\title{
Power System Load Modeling Based on Genetic Programming
}

\author{
Jian Zhang \\ Zhengzhou University \\ Zhengzhou Henan China \\ 2275538863@qq.com
}

\author{
Chaohui Zhang \\ Zhengzhou University \\ Zhengzhou Henan China \\ 364903034@qq.com
}

\begin{abstract}
Genetic Programming (GP) is a new evolutionary algorithm based on genetic algorithm, which has selfadaptive, self-organizing, self-learning and other advantages, and has significant advantages in terms of symbolic regression to solve long-term problems of the model structure automatically recognizes the problem. In this paper, the genetic programming is introduced to the power system load modeling to solve long-standing problems of automatic identification model structure in the power system.
\end{abstract}

Keywords- Load Model; Genetic Programming; Model Structure; Model Identification; Simulation

\section{INTRODUCTION}

Digital simulation of power systems has become an important tool of the planning, design, scheduling power system operation and experimental study. The simulation results are accurate or not directly determine the power sector decision-making in these areas correctness, and the accuracy of the simulation model used to direct effect of simulation results. In recent years, modeling technology of generators and control systems and transmission network has become mature. Their model structure can accurately reflect the actual operating characteristics. By contrast, due to the randomness, complexity, distributed and other characteristics of the power system load, accurate load modeling has been difficult [1]. The existed modeling method is difficult to accurately describe the actual power system model, impacting the accuracy of digital simulation .Genetic programming (referred as GP) is a new evolutionary algorithm developed on the basis of genetic algorithms in the 1990s, (genetic algorithm, referred to as GA).Currently, genetic programming has become an important branch of the evolutionary algorithm and it has successfully been applied to pattern recognition, function regression, economic forecasts, and other fields. This paper attempts to introduce the GP model structure to build a power system load, the use of GP characteristics in terms of function mining to solve long-term problems of model structure automatic identification in load modeling.

\section{STUDY OF POWER SYSTEM LOAD MODELING}

The load characteristic of power system refers to the active power $\mathrm{P}$ and reactive power $\mathrm{Q}$ flows into the load changes with the voltage and the system of frequency, and it can be divided into voltage and frequency characteristics of the active power $\mathrm{P}$ and the reactive power $\mathrm{Q}$, the mathematical expression used to describe the load characteristics is load model. Typically, the frequency fluctuation of power system is not big, therefore, in some occasion where is less demanding on the accuracy, the effect of frequency fluctuation on system load can be ignored [2]. In this paper, the main consideration is the impact of voltage change on the system power.

\section{A. Load modeling method of commonly used [3]}

After the long-term research and development, currently there are the following three kinds of commonly used methods of load modeling in power system:

1) Component-based modeling approach

This modeling approach is by testing a variety of typical system load originals (such as electric motors, air conditioning, incandescent, etc.) to deduced a mathematical model and stats the proportion of each typical load in integrated load, finally get the load model by consolidating these dates.

\section{2) Measurement-based modeling approach}

The method is developed along with the development of computer and evolutionary algorithm. It puts the load as a whole, based on the measured data collection, using artificial evolution of computer code to identify the structural load model, and then through some algorithm for parameter optimization, load model is obtained.

\section{3) Fault-based modeling approach}

This method firstly choose a certain common model (such as ZIP model, power function model) by the staff according to their own experience, operation features of the system and the load's basic composition, using the least square method and other mathematical methods to identify model parameters, and then constantly adjust the model structure and parameters through the simulation and comparison of system response curve obtained from test , and finally make the simulation results to approach the real response curves of the system.

\section{B. The shortcomings of the current modeling technology}

The so-called load model includes two parts: model structure and model parameters. The model structure is the morphology of a model, and largely reflects the nature of the load. If choose the wrong model structure, even if identify the best model parameters, the model can't correctly describe the character of load. The identification of model structure for a long time is the key of the load modeling work, but also the most immature and difficult places in research. And the determination of model parameters is relatively mature, relatively easy to 
determine. Load model is decided basically by the modeler for specific load object. According to the experience of artificial selection for model structure, the selection always has a very big subjectivity. In the face of tens of thousands of possible combination for model structure scheme, it is difficult to select optimization model structure by manual methods [4]. Therefore, research on the optimized model structure established by computer, without human intervention or less dependent on experiential knowledge is of great importance.

Using genetic programming based on evolutionary computation to load modeling, there is no need to predetermine the model structure. It can reach the function relation of the variables by evolutionary computation of the input dates, which provides a new way for electric load computer automatic modeling.

So far, there has not been a study of power load modeling using the methods reported and this is also the innovation of this paper.

\section{THE BASIC IDEA OF GENETIC PROGRAMMING}

Genetic programming inherits the basic idea of genetic algorithm (GA), the essential difference between GP and the genetic algorithm is the difference of the genetic individual expression in population. Genetic algorithm uses fixed length genome bit string, while the GP uses the genome bit string of variable length tree data structure which adopts a hierarchical structure expression. GP uses natural automatic selection principle and the theory of biological evolution, with the method of "survival of the fittest", starting from a randomly generated initial population, measuring individual advantages and disadvantages by fitness, the fitness of individuals directly copied into the next generation [5].If individuals' fitness is not good, through the cross, mutation, recombination and other operations change the individual structure. After several generations of evolution it adapts to the best individual and that is the evolution of structural optimal model. The main program flow chart of GP is shown the fig.1.

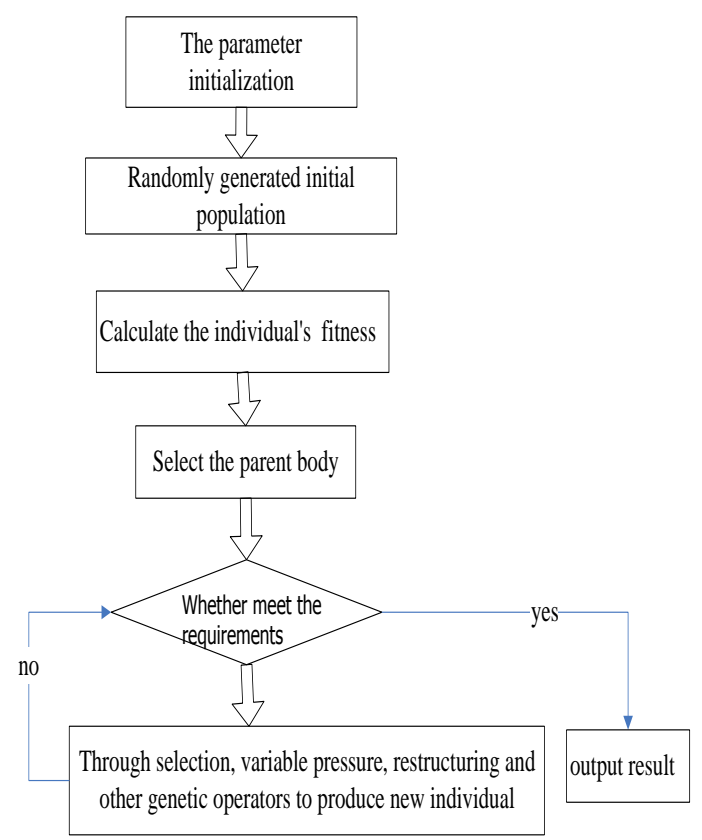

Figure 1. The main program flow chart of genetic programming

\section{A. Function representation}

Function expression in GP model is represented by using dynamic tree structure which is called tree algorithm. Non leaf node of tree algorithm is constituted by the function symbol, the leaf nodes by the terminator. The function symbol set can be arithmetic, Boolean operators, conditional expression and any function can be defined Termination character set can be a variable or constant. The representation of tree algorithm in the computer is a decimal shaping code string. The first half of code strings are non- leaf nodes, and the latter part is the leaf node.The location of the code is equal with the node location in the tree algorithm ,the value of code corresponding to the elements of the function set and termination set[6]. The algorithm tree structure is shown in fig. 2 . Take into account the convergence and operability of the algorithm the function symbol set used in this paper is symbols $\{1\}=\left\{\right.$ ' $^{\prime},{ }^{\prime}-{ }^{\prime},{ }^{\prime} \cdot{ }^{*}, ' . /$ ' $\}$, and termination symbol set is symbols $\{2\}=\left\{\right.$ 'x' $\left.^{\prime}\right\}$.

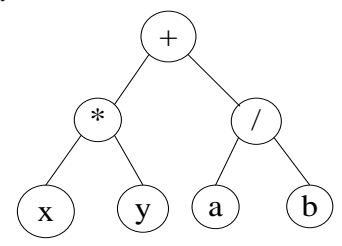

Figure 2. Tree algorithm

\section{B. Fitness selection and shutdown condition}

Evolutionary modeling is to get the fitting error small, and the model structure expression clear. Therefore it is necessary to evaluate the individual merit which is obtained by evolving, fitness is the evaluation of individual quality standards [7]. This paper uses the errors of least squares method as the individual fitness value. The $f\left(x_{i}\right)$

variable data $\mathrm{J}$ in a sample of values. The ${ }^{y_{i}}$ represents the actual measurement values of samples, then the fitness of individuals for:

$$
\operatorname{Fit}\left[f\left(x_{j}\right)\right]=\sum_{j=1}\left[f\left(x_{(i, j)}-y_{j}\right]^{2}\right.
$$

Obviously, the smaller is the individual fitness, the better is the function model generated.

Generally there are two stop conditions: One is when the evolution of maximum permissible evolution algebra satisfies the conditions. The other one is when the evolutionary individuals adapt to the evolution degree requirements. This paper uses the method provides evolutionary iterations when subjected to a prescribed number of iterations the evolution stops, choose the best individual with best fitness.

\section{The choice of genetic operator}

In order to guarantee the convergence of genetic programming, it needs to take manipulation on the individuals during the process of evolution. This paper adopts the following three kinds of genetic operator [8]. 
The selection operator: Copy the individuals of good fitness into the next generation directly without any change, which embodies the superior principles during the process of evolution.

Crossover operator: (As shown in Fig. 3)In any two of the parent body, choose two binary tree nodes as independently hybridization, then, exchange the corresponding sub-tree which focus on the hybrid of the parent body, get two generations. In order to avoid the complicated model introducing a parameter called the maximum depth. Make the depth of the two fork tree in the model less than the depth parameter. In this paper the biggest tree in the deep set to 7 .

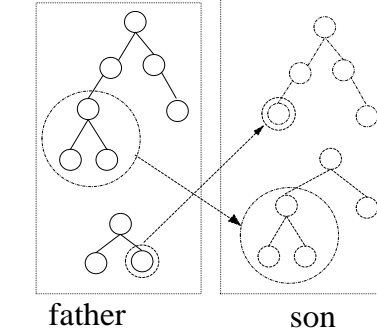

Figure 3. hybrid operation

Mutation operator: (As shown in Fig. 4)Select any one individual tree, in this algorithm, randomly selected a node tree as the mutation point. This could be as a function point, also can be a terminator. Then delete the mutated point and the sub-tree below it, choosing a new sub-tree by random and insert mutation point, then generate a new tree [9].

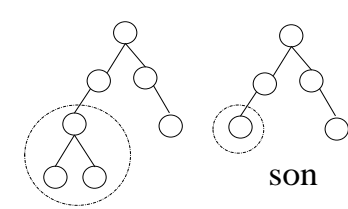

father

Figure 4. Variation

IV. EXAMPLES OF CALCULATION AND ANALYSIS

The data used in this experiment was obtained in the lab when testing static voltage characteristic for induction motor. (As shown in Table 1)

TABLE I. RESULTS OF ACTIVE AND REACTIVE POWER LOAD CHARACTERISTIC TEST (TABLE DATA ARE NORMALIZED VALUE)

\begin{tabular}{c|c|c}
\hline $\mathrm{U}$ & $\mathrm{P}$ & $\mathrm{Q}$ \\
\hline 1.121053 & 0.484515 & 0.922466 \\
\hline 1.052632 & 0.428333 & 0.736968 \\
\hline 1.000000 & 0.41278 & 0.630898 \\
\hline 0.947368 & 0.403545 & 0.531095 \\
\hline 0.905263 & 0.400569 & 0.465205 \\
\hline 0.852632 & 0.386055 & 0.404971 \\
\hline 0.800000 & 0.386707 & 0.335861 \\
\hline 0.747368 & 0.380415 & 0.285312 \\
\hline 0.705263 & 0.375948 & 0.252639 \\
\hline 0.652632 & 0.373822 & 0.216848 \\
\hline 0.600000 & 0.374814 & 0.181531 \\
\hline 0.547368 & 0.367592 & 0.167480 \\
\hline 0.526316 & 0.366922 & 0.161788 \\
\hline 0.505263 & 0.371988 & 0.157905
\end{tabular}

\begin{tabular}{l|l|l}
\hline 0.484211 & 0.376991 & 0.154859 \\
\hline 0.473684 & 0.382937 & 0.154340 \\
\hline 0.463158 & 0.378579 & 0.157830 \\
\hline 0.452632 & 0.377283 & 0.160153 \\
\hline 0.442105 & 0.374799 & 0.159644 \\
\hline 0.431579 & 0.375938 & 0.162969 \\
\hline 0.421053 & 0.382451 & 0.170892 \\
\hline 0.410526 & 0.380307 & 0.170439 \\
\hline 0.400000 & 0.381883 & 0.173991 \\
\hline 0.389474 & 0.383498 & 0.175285 \\
\hline 0.378947 & 0.378111 & 0.179902 \\
\hline 0.368421 & 0.395578 & 0.210816 \\
\hline 0.357895 & 0.411276 & 0.241309 \\
\hline 0.347368 & 0.421358 & 0.272170 \\
\hline
\end{tabular}

A. Experimental parameters design and experimental results

The programming language and environment: matlab 7.0. Define a function symbol set is symbols $=\left\{{ }^{\prime}+{ }^{\prime},-{ }^{\prime},{ }^{\prime}{ }^{\prime}, '\right.$. /'\}. Maximum tree depth maxtreedepth $=7$, iterations popusize $=5000$, to determine the optimal model structure by using the least square method to calculate the minimum error sum of squares.

The optimal model structure is obtained after 5000 iterations shown as follows:

$$
\left\{\begin{array}{l}
(x) \cdot *((x) \cdot /((((x) \cdot *(x))+((x) . /(x))) \cdot /(x)) \cdot /(x))) \\
((x) \cdot *(x))+(x)
\end{array}\right.
$$

\section{B. Simulation results}

Suppose the load model is a power function model. According to the data in Table 1, the model of the power function get by using evolutionary programming is [10]

$$
\left\{\begin{array}{l}
P=0.407 U^{0.074} \\
Q=0.58 U^{1.39}
\end{array}\right.
$$

Fit the experimental dates use five polynomial fitting by least square method, and then, compare these two results with the model build with GP, we can get the following results just as the figure 5 and figure 6 .

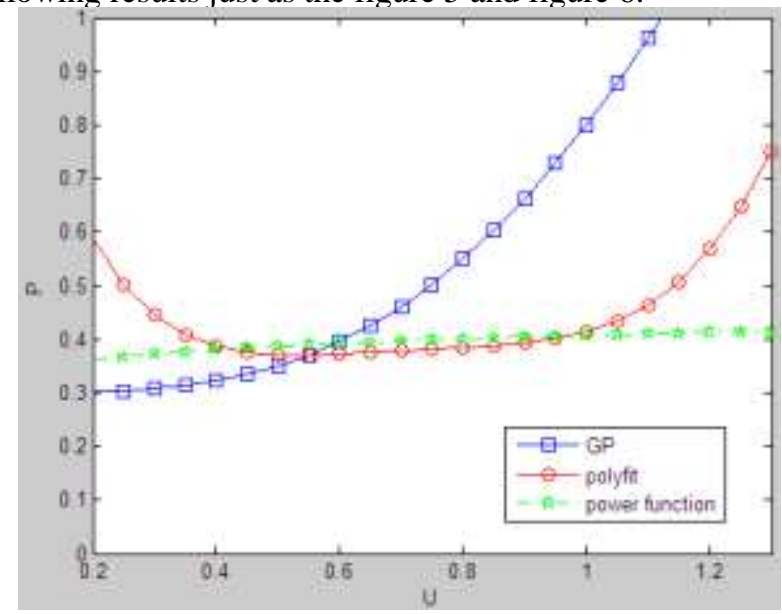


Figure 5. Active load characteristic curve

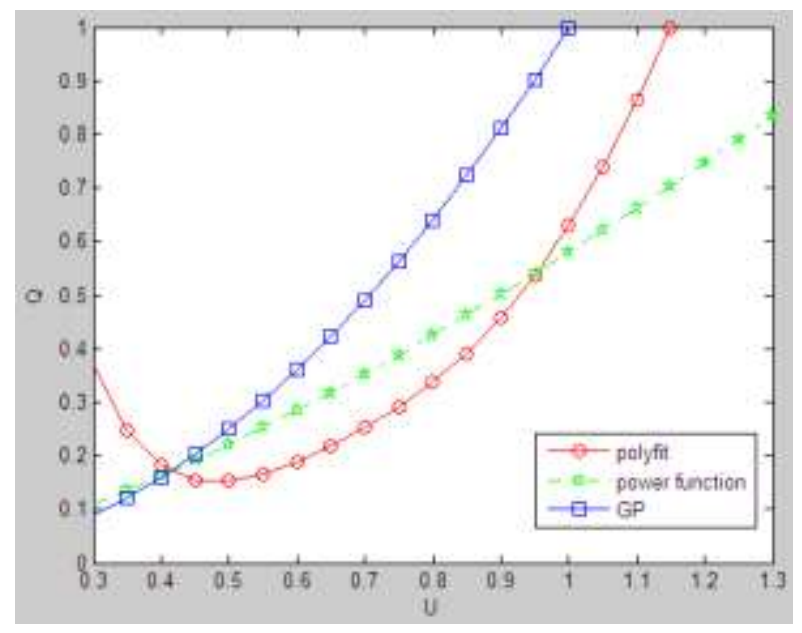

Figure 6. Reactive load characteristic curve

\section{Analysis of the results}

From the results above we may see, when using the genetic programming to electric load modeling, through proper design of experimental parameters can get the flexible function structure, and get the function relationship of the internal datas automatically, find the better model structure better in the short run time and the evolutional gebra, the model structure build up by this method can get better fitting effect too. Especially when the function structure are looking for is more complex, it can also find the internal relations and rules between dates which are hard to find by artifical. Break through the limitations of relying too heavily on artificial experiences of load modeling project in the power system.

\section{CONCLUSIONS}

This paper has realized the automatic establishment of load model by using genetic programming and carried on the simulation, but there are still some problems, such as the fitting precision is not very high. The next step will be considered for model structure identification by using optimization genetic programming, identification of parameters by the genetic algorithm or the traditional optimization algorithm, and then establish the more accurate model structure and realize the automatic load modeling of power system.

\section{REFERENCES}

[1] Zhang Jian, the load model of power system and the identification of [M].Beijing: China electric power press, 2007

[2] Zhou Wen, He Renmu, Zhang Jian, Li Xinran. Review of electric load modeling research [J]. Modern electric power.1999, 16 (2).8389

[3] IEEE Task Force on Load Representation for Dynamic Performance Standard load models for power flow and dynamic performance simulation $[\mathrm{J}]$. IEEE Trans on Power Systems, 1995, 10(3) : 1302-1313.

[4] He Renmu, Wang Weigong, Jiang Debin, et al. Measurementbased dynamic load modeling and model validation on Guangdong Grid $[\mathrm{J}]$. Proceedings of the CSEE, 2002, 22 (3) : 78-82(in Chinese) .

[5] Liu PU. Evolutionary algorithm and its parallel implementation. Doctoral dissertation of Wuhan University, 2000

[6] Holland John H. Adaptation in Natural and Artificial Systems [M].Ann Arbor: University of Michigan Press, 1975.

[7] KANG Lishan, LI Yan, CHEN Yuping. A Tentative Research on complexity of Automatic Programming [J]. Wuhan University Journal of Natural Science, 2001, 6(1-2): 59-62.

[8] Ricardo Alert, Daniel Borrego, Pedro Isasi. Using genetic programming to learn and improve control knowledge [J]. Artificial Intelligence, 2002, 141(1 - 2):29 - 56.

[9] Douglas B. Kell. Genotype2phenotype mapping: genes as computer programs [J]. Trends in Genetics, 2002, 18(11):555 - 559.

[10] Mark P. Hinchliffe, Mark J . Willis. Dynamic systems modeling using genetic programming $[\mathrm{J}]$. Computers \&Chemical Engineering, 2003, 27(12 - 15):1841 - 1854. 